\title{
Perceived Health Status of Women with Knee Osteoarthritis: A Cross- Sectional Study of the Relationships of Age, Body Mass, Pain and Walking Limitations
}

\author{
R. Marks*
}

School of Health \& Behavioral Sciences, City University of New York, York College, and Department of Health \& Behavior Studies, Columbia University, Teachers College, New York, USA

\begin{abstract}
Persons with knee osteoarthritis (OA) often experience considerable physical disability. Although some studies suggest women with this condition suffer more than men, few have attempted to characterize the magnitude and that impact of this condition specifically among women with moderate knee osteoarthritis as well as the relationships that exist between their perceived health status and well established physical, emotional and perceptual factors found in this disease. This exploratory study strove to better understand factors that underpin the perceived impact of the condition, and to describe the extent of pain and function among women with mild to moderate knee osteoarthritis, and how this impacts this condition. The records of 20 women with the condition who had undergone multiple tests using a standardized protocols and validated instruments were examined. The primary outcome measure was the perceived impact of the disease using the Arthritis Impact Measurement Scale. Secondary outcome measures included six minute walking distance, fastest walking velocity, self-reported pain, pain and functional self-efficacy, body mass, and depression. The variables were subjected to t-tests, and correlational analyses. Results demonstrated pain is the clinical factor most consistently impacting the disease experience, along with deficiencies in walking ability $(\mathrm{p}<0.05)$. Important mediating variables of ambulatory capacity were body mass and pain self-efficacy.
\end{abstract}

Keywords: Depression, function, knee, osteoarthritis, pain, self-efficacy, walking.

\section{INTRODUCTION}

Osteoarthritis (OA), a prevalent joint disease causes appreciable disability in the majority of adults over the age of 55 [1]. At the knee, a joint frequently affected by the disease $[2,3]$, the disease produces considerable functional disability and pain [4], especially in women [5, 6]. Studies have revealed that compared to healthy women, women with knee osteoarthritis have decreased survival rates [7], and that as a whole, its individual and collective socio-economic impacts [8] as a result of multiple pathological features [9$13]$, are considerable $[14,15]$.

Moreover, this series of generally irreversible pathological processes [16], can be accompanied by episodes of intractable pain, joint swelling, and stiffness, limitations of joint range of motion, muscle strength, and the efficiency of musculoskeletal system [17]. The chronicity of the disease can also produce emotional distress, depression and/or impairments of general health and vitality, reduced life quality, activity limitations [16], negative personal beliefs, as well as sleep disturbances. These changes and others such as poor cardiovascular endurance, low exercise tolerance $[17$, 18], and diminished ability to function physically [19] without undue exertion [20] can also increase the risk for

*Address correspondence to this author at the School of Health \& Behavioral Sciences, City University of New York, York College, and Department of Health \& Behavior Studies, Columbia University, Teachers College, New York, USA; Tel: 718-262-5109; Fax: 718-262-5216;

E-mail: rmarks@york.cuny.edu other health problems such as obesity. The disease can severely impair the function of the knee extensor muscles $[14,21,22]$, and in longstanding cases, muscle atrophy of both fiber types is likely to ensue, thus further impairing life quality [21], joint stability [23] and functional capacity [23].

However, even though numerous studies conducted in the last 30 years have examined some of the aforementioned factors in isolation, very few have focused on examining the interaction of the perceptions of patients with this condition, including their estimation of its impact and the extent to which pain and feelings of depression, health, disease duration, and body weight status contribute to this perception. Moreover, in studies that do exist, most have included patients with hip osteoarthritis and have assessed men and women and their disability expressions simultaneously even though the etiology may well differ in some respects between genders [22].

Thus, which factors best explain the overall impact of knee osteoarthritis on the day to day wellbeing of women with knee osteoarthritis, who are commonly affected more than men [22] is not clear and has been very poorly studied [16]. In addition, by including patients younger than 60 years of age, several prior studies conducted among patients with knee osteoarthritis have not been able to clearly clarify the impact of living with this disease among the older adult. Nor has the impact of aging, as well as the impact of the disease among women with knee osteoarthritis been well examined in a single context. Finally, whether cardiovascular or other comorbid diseases produce excess disability and impairment 
in women with this disorder is not well reported even though cardiovascular risk factors are found at higher rates in the rheumatological diseases than in control populations [24].

The specific objective of this research was to examine some key variables associated with the perceived impact of knee joint osteoarthritis among a small group of women 60 years and older with radiological and clinical verification of unilateral or bilateral knee joint osteoarthritis living in the community. The women were eligible to enter a study to examine the efficacy of an exercise protocol for improving their knee osteoarthritis. In addition to measuring the impact of the condition, an accepted proxy for health status, several well-established physical and psychological correlates of knee osteoarthritis were specifically examined to determine their relative importance as regards improving the health status of this group [16].

It was hypothesized that the women examined would show significant relationships between their walking ability, and their perceived health status and that this would be impacted by both age and pain. It was also believed that pain would be a strong explanatory variable of the impact of the disease, as would body mass and self-perceptions of confidence to carry out arthritis related management approaches

It was anticipated that the results of this study could help to inform clinicians about what remedial efforts might be especially valuable for enhancing functional independence and health related quality of life in women with similar personal characteristics.

The specific questions of interest were:

1. What are the key personal, physical, and health characteristics of women 60 years and older with radiographic and clinical evidence of knee osteoarthritis?

2. What are the main features of the condition that appear to be subject to change according to decade of age?

3. What are the factors that best correlate with the patients' perception of the impact of the disease on their wellbeing?

4. What can the data reveal that will help advance recommendations for reducing the risk of excessive disability among a similar group of adults?

\section{MATERIALS AND METHODS}

An available sub-sample of 20 subjects drawn from a larger study fulfilling the inclusion criteria for the present study was conducted. Eligible subjects were examined as regards disease presentation, duration, pain, medical, functional and cognitive status on a single test occasion. Subjects were women with radiological evidence of knee osteoarthritis of either the medial or lateral compartment of one or both knees of grade II severity (i.e. joint space narrowing, osteophytes) recorded in weightbearing [5]. All subjects had to be able to walk with or without an aid and present with at least 3 of 6 criteria [25] as follows:

Age $\geq 60$ years.
- $\quad$ Morning stiffness $<30$ minutes.

- $\quad$ Crepitus on knee motion.

- Bony tenderness knee joint margins.

- Bony enlargement knee.

- $\quad$ No palpable warmth.

Excluded were:

- $\quad$ Adults with rheumatoid arthritis, or definitive arthritis of other joints.

- Men.

- $\quad$ Adults with evidence of neurological impairment.

- Adults with evidence of limb injury or abnormality.

- Women with knee osteoarthritis eligible for surgery or a history of surgery of the affected knee [s].

- Women with knee osteoarthritis presenting with uncontrollable or unstable medical conditions.

- Those unable to follow instructions or basic English.

Subjects were referred to the study by rheumatologists at the University Hospital. All decisions regarding subject suitability for the study were made by the investigator. All subjects were required to give informed consent.

To obtain the desired data, several self-reports were completed by eligible participants under the same conditions in a single hospital setting. These tests included a number of validated surveys including: The Arthritis Impact Measurement Scale (AIMS)(Physical, Health, Pain and Impact Subscales) [26], The Center for Epidemiological Studies Depression Scale (CES-D) [27], a $10 \mathrm{~cm}$ long visual analogue pain scale where 0 was no pain [28], The Borg Perception of Effort Scale [29], and The Arthritis SelfEfficacy for Pain and Other Symptoms Scales [30].

Demographic variables captured included age, education, marital status, numbers of affected joints, health status, height, and weight. Self-reported pain was also assessed using the 4 pain and stiffness questions on the Arthritis Impact Measurement Scale that was scored as a composite variable by collapsing the 4 scores according to standard procedures and where overall scores range from 1-6, with 1 being indicative of more severe disability, and 6 minimal disability, respectively. Medical history and arthritis impact (0-100 visual analogue scale) were documented as per the AIMS check list. Standard body weight $(\mathrm{kg})$ and height $(\mathrm{m})$ measures were used to derive a body mass index $\left(\mathrm{w} . \mathrm{h}^{-2}\right)$ for each individual. Depression using the CES-D was estimated by summing the 20 -item scores, which could range from 0 60 , with a cut-off point for depression of 16 .

Total distance walked in 6 minutes at self-paced speed on a standardized level indoor walkway and what factor [s] most commonly prevented or limited ambulatory performance was measured using a checklist of 4 options (shortness of breath, pain, fatigue, other). Perceived exertion at the end of a six minute walking test on level ground, where individuals were encouraged to walk as far as possible was measured on a visual analogue scale ranging from $0-15$, where 0 was no exertion and 15 maximal exertion. Functional performance was also assessed through a self- 
selected slow and fast walking speed test that was recorded in $\mathrm{m} . \mathrm{s}^{-1}$ on a level indoor walkway using an electronic timer and floor markers and measuring the duration of the gait cycle. Electronic records of how the individuals compared to healthy age matched norms as regards the gait cycle were calculated for each participant.

In addition to documenting the mean age, and disease duration of the cohort, several subgroup analyses were conducted for the variables of age (60-69, 70-79, 80+ years); cardiovascular history (presence of versus no cardiovascular disease history; cardiac disease versus hypertension versus no disease); osteoarthritis status (unilateral versus bilateral knee joint disease); depression (presence of depression versus no depression) and normal weight versus overweight and obese categories of patients.

The data were entered systematically into an Excel spreadsheet by the researcher, and the desired descriptive statistics, and inferential tests including analysis of variance, regression analysis and selected correlations were calculated using SPSS version 16.00. The prevalence of comorbid conditions and their diagnostic labels, plus demographic and disease descriptors were noted and reported together and separately for 3 age categories $(60-69 ; 70-79 ; 80+$ years) to examine the effects of aging on disease correlates. Cognitive status and perceptions concerning personal efficacy for managing pain and other symptoms were recorded as well. Correlations were generated to evaluate the influence of changeable factors such as pain, perceived impact and emotional status with ambulatory function indicators, in addition to age. All significant variables were entered together as independent variables in a stepwise regression model to estimate the most salient relationships of variables with a correlation significance of $p=0.20$ or lower with respect to arthritis impact scores, AIMS and visual analogue pain scores, and six minute walking distance. The significance level was set at $p<0.05$. The separate regression analyses conducted on all potential predictors included correlation and partial correlation statistics, and all standard diagnostic procedures. A power analysis conducted prior to study showed a minimum of 17 subjects would be needed to demonstrate significant interrelationships between arthritis impact and pain and function. Twenty subjects were recruited to allow for dropouts and any missing data.

\section{RESULTS}

Of the 20 women recruited, 17 had complete records, and among these, 14 were Caucasians, 2 were Non-Hispanic Blacks, and one was Hispanic. These women ranged in age from 60 to 84 years with a mean age of $70.8 \pm 7.5$ years. Seven were college graduates, five had some college exposure, and with the exception of one subject, the rest were high school graduates (See Table 1). Only 2 were presently married. The rest were either widows, divorced or unmarried. As outlined in Table 1, which portrays the mean and range of scores recorded for the present group as regards variables of interest, only $18 \%$ of the present cohort could be categorized as being in the normal weight range. Eight had unilateral knee joint osteoarthritis, and nine had bilateral knee joint osteoarthritis, but there were no differences in AIMS pain scores $(\mathrm{p}=0.988)$, disease duration $(\mathrm{p}=0.678)$, walking distance scores $(\mathrm{p}=0.384)$, velocity of fast walking $(p=0.110)$ or age $(p=0.456)$ between those with unilateral and those with bilateral joint disease using analysis of variance. Of the 17 cases assessed presently, 12 stated they were in good health, and two stated they were either in excellent or fair health, even though $11(65 \%)$ had a history of one or more cardiovascular conditions. Three however, exhibited evidence of depression of moderate severity, and this condition was found to be more prevalent in the oldest age category (see Table $\mathbf{2}$ ).

When examined more closely in terms of a breakdown in age range, Table 2 also shows a decline in fast walking velocity and walking distance in the highest age range, where numbers of medical conditions were higher. Pain was also experienced more intensely in those who were older than 69 years of age. Arthritis impact scores, a proxy for health status, as well as physical disability scores were also higher in the highest age group, as was pain, but these finding were not significant.

The general characteristics of those with and without cardiovascular disease histories, which was of interest in the context of this exploratory analysis is shown in Table 3. These data suggest that those with a cardiovascular history may be more prone to depression and lower perceptions of self-efficacy for pain and other symptom management. Interestingly, those with no cardiovascular disease were significantly heavier than those with one or more cardiovascular diagnoses, suggesting that they may be prone to cardiovascular disease later on or that obesity itself produces as much disability in otherwise healthy people with knee osteoarthritis, as those with one or more cardiovascular conditions. Very few other health conditions were noted except for one case of diabetes. The subjects with cardiovascular diagnoses generally reported higher AIMS Impact scores than those with high blood pressure diagnoses ( 5.8 versus 2.9 , and denoting poorer health status), they had more pain on average (AIMS Pain score 5.5 versus 4.6), poorer physical function (AIMS Physical 7.3 versus 4.8 ) and walking speed (48.9 versus $\left.56.6 \mathrm{~m} . \mathrm{s}^{-1}\right)$ than those with high blood pressure, but this was not significant.

In terms of relationships among the variables analyzed, there were several noteworthy bivariate correlations. These occurred between the variables of Arthritis Impact and pain scores and between pain and walking ability as outlined in Table 4. Among some of these correlations, age was related to fast walking speed $(\mathrm{r}=-0.48, \mathrm{p}=0.052)$ suggesting those who are older walk more slowly than those who are younger. In addition, perceived exertion when walking was related to self-reported pain $(\mathrm{r}=0.50, \mathrm{p}=0.043)$, suggesting perceived exertion may limit function as may bodyweight because walking distance was inversely related to body mass index $(\mathrm{r}=-0.42, \mathrm{p}=0.051)$.

Another finding was that the higher the impact of the disease, the slower the fast walking velocity as depicted by partial correlations of $-0.48, p=0.050$. Finally, depression scores were negatively associated with walking distance (partial correlation $\mathrm{r}=-0.25)$, pain self-efficacy $(\mathrm{r}=-0.75$, $\mathrm{p}=0.001)$ and self-efficacy for function $(\mathrm{r}=-0.56, \mathrm{p}=0.013)$, suggesting those who are depressed may not be able to walk as far in six minutes as those without depression, and either 
Table 1. Descriptive characteristics of sample, ages 60 years and older with unilateral or bilateral knee osteoarthritis, randomly selected from 47 eligible participants with complete records means, range and percentage distribution of key variables $(\mathrm{N}=17)$.

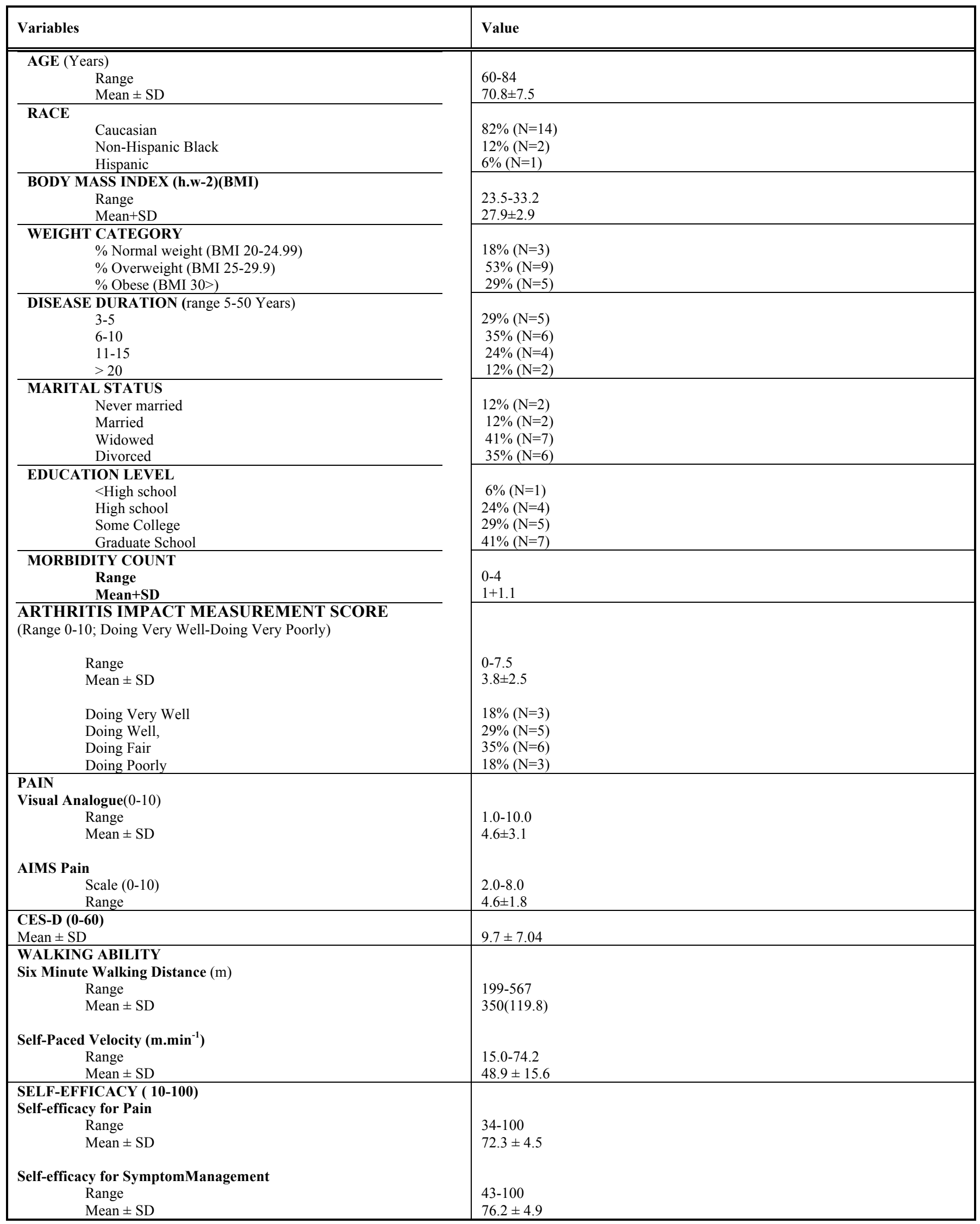


Table 2. Prevalence of ambulatory disability by age grouping showing impact of age on walking ability, pain and medical profile using univariate analysis.

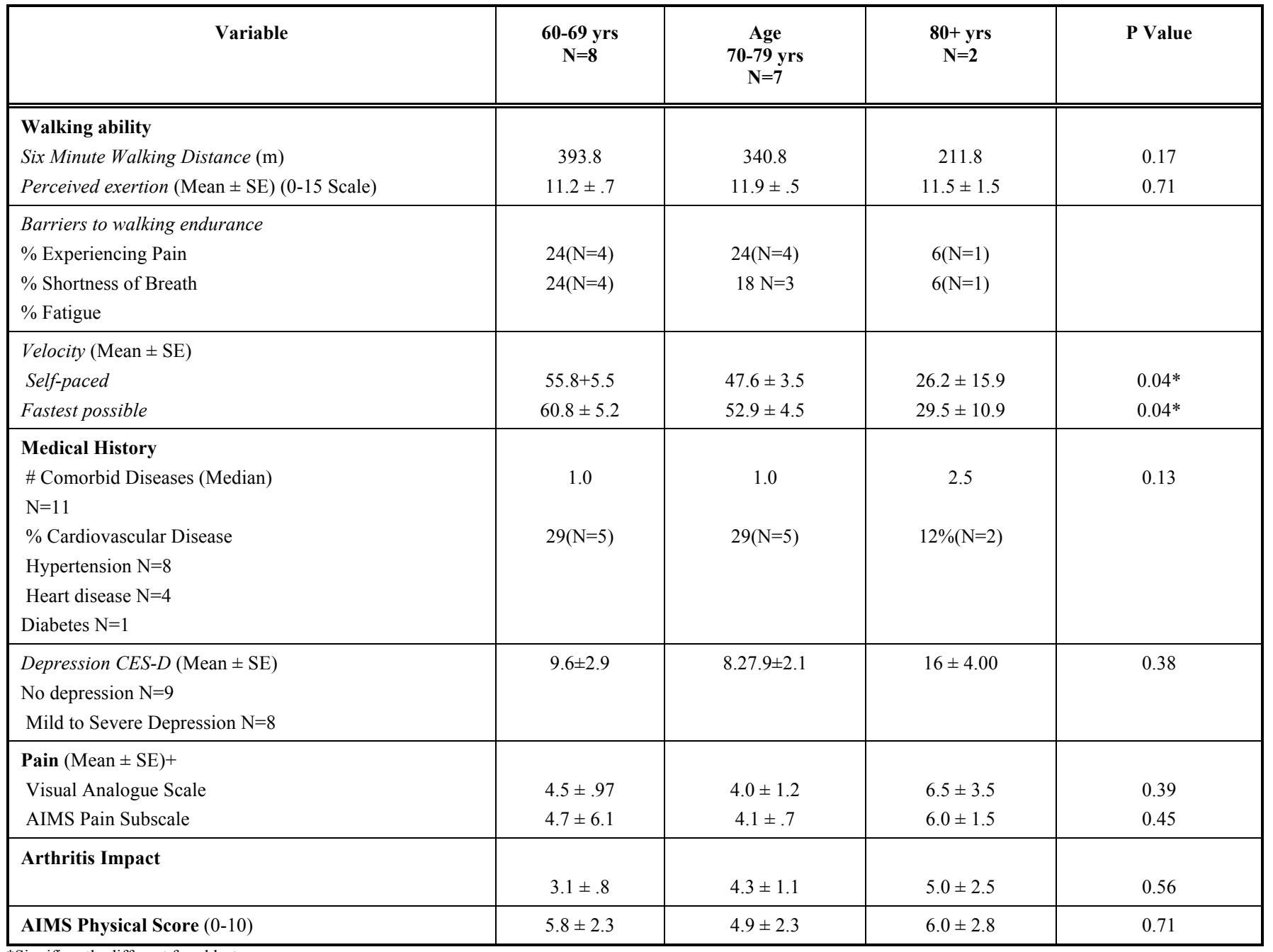

*Significantly different for oldest group.

do not perceive they can function or manage their disease as well as others or their perceptions of their abilities augment depressive tendencies. In terms of overall walking ability, the average time spent in a single gait cycle of $1.32 \pm 0.1 \mathrm{~s}$ was consistently longer in duration than anticipated among healthy women of similar ages in 100 percent of cases. This walking pace of $30 \%$ or less than the average walking speeds of healthy women, was correlated with the patients perception of the impact of arthritis on their wellbeing.

However, among the variables identified in Tables $\mathbf{4}$ and 5, stepwise regression showed the best predictor of Arthritis Impact was the AIMS pain score $(p=0.021)$, which was positively correlated with this with a partial correlation of $\mathrm{r}=0.56$, beta $=0.56$. The next strongest explanatory variable was self-paced velocity, partial correlation $r=-0.46, p=0.077$, beta $=-0.40$ followed by six minute walking distance $(\mathrm{r}=-0.42, \mathrm{p}=0.103$, beta $=-0.36)$ and fast walking velocity $(\mathrm{r}=-0.37, \mathrm{p}=0.159$, beta $=-0.33)$. Although Arthritis Impact mean values increased in higher age groups shown in Table 2, age was a weak predictor of Arthritis Impact $(r=0.30$, $\mathrm{p}=0.267$, beta $=0.25$ ).
In additional regression analyses, the best predictor of the AIMS pain score was the overall pain experience of the subject as measured on the visual analogue scale $(\mathrm{r}=0.79$, $\mathrm{p}=0.001$, beta $=0.79$ ), which was associated most strongly with perceived exertion after walking for six minutes $(r=0.50, p=0.043$, beta $=0.50)$, followed by body mass index $(\mathrm{r}=0.28, \mathrm{p}=0.290$, beta $=0.25)$. Best predictors among the zero order correlations related to six minute walking distance were self-paced walking velocity $(r=0.83, \quad p=0.001$, beta $=0.83)$ followed by body mass index $(r=-0.59, p=0.015$, beta $=-0.34)$. The interrelationship of these key factors is portrayed in Fig. (1).

\section{DISCUSSION AND CONCLUSION}

Osteoarthritis, an age-related disease of the articular cartilage lining of a joint, produces a multi-dimensional clinical syndrome, which often includes symptoms of pain. Associated pathological changes such as joint effusion, stiffness, decreased range of joint motion, joint instability and malalignment are common, and can progressively impair the efficiency of musculoskeletal functioning in this population $[18,19]$. 
Table 3. Summary of measures and univariate analysis of age, disease duration, body mass, function and arthritis impact by cardiovascular status (Mean $\pm \mathrm{SE}$ ) $[\mathrm{CI}]$.

\begin{tabular}{|c|c|c|c|}
\hline Variable & No Cardiovascular Disease $N=6$ & Cardiovascular Disease $\mathrm{N}=11$ & $\mathbf{P}$ \\
\hline Age (years) & $69.5+2.4[63.3-75.7]$ & $71.5+2.5[65.8-77.1]$ & 0.62 \\
\hline AIMS Physical Score (0-10) & $4.7 \pm 0.7[2.95-6.4]$ & $5.8 \pm 0.7[4.18-7.5]$ & 0.32 \\
\hline AIMS Pain (0-6) & $4.6 \pm 0.7[2.7-6.5]$ & $4.6 \pm 0.6[3.4-5.9]$ & 0.95 \\
\hline Visual analogue pain score $(0-10)$ & $4.8 \pm 1.7[0.56-9.1]$ & $4.4 \pm 0.8[2.7-6.2]$ & 0.80 \\
\hline CES-D & $6.3 \pm 1.1[3.6-9.04]$ & $11.6 \pm 2.4[6.1-16.98]$ & 0.15 \\
\hline BMI $\left(h \cdot w^{-2}\right)$ & $29.8 \pm 1.4[26.4-33.3]$ & $26.9 \pm 0.6[25.5-28.3]$ & $0.03^{*}$ \\
\hline Pain Self Efficacy (10-100) & $78.3 \pm 6.1[62.7-94.0]$ & $69.1 \pm 6.0[55.7-82.5]$ & 0.34 \\
\hline 6 min Walking Distance $(\mathrm{m})$ & $321.7+49.3[195.0-448.7]$ & $366.2 \pm 38.6[280.3-452.2$ & 0.49 \\
\hline Perceived Exertion $(0-15) *<0.05$ & $11.3 \pm 0.7[9.5-13.2]$ & $11.6 \pm 0.5[10.5-12.6]$ & 0.81 \\
\hline
\end{tabular}

$*<0.05$.

Table 4. Significant bivariate zero order correlations observed between aims impact scores and key functional and cognitive measures $(\mathrm{N}=18)$.

\begin{tabular}{|c|c|c|c|c|c|c|}
\hline AIMS Impact & WlkDist & SPVel & Gait Cycle & VAS Pain & AIMS Pain & Pain SE \\
\hline \hline $\mathrm{r}$ & -0.53 & -0.60 & 0.54 & 0.50 & 0.53 & -0.47 \\
$\mathrm{P}$ & 0.024 & 0.008 & 0.032 & 0.036 & 0.025 & 0.048 \\
\hline
\end{tabular}

Abbreviations: AIMS Impact=Arthritis Impact (0-10); Pain SE=Pain self-efficacy scores; SPVel=Self-paced walking velocity; VAS=visual analogue pain scale scores (0-10); WlkDist $=6$ minute walking distance.

At the knee, the most commonly involved major joint affected by OA [3], muscle weakness, pain and functional impairment prevail and are magnified as the condition worsens, especially among women [6]. Less well documented are changes in general health status, the role of obesity in the disease process, the role of aging, cognitions and vascular health that may contribute to the ambulatory limitations commonly experienced by this patient group, and by women who tend to suffer more from this condition than men.

The present exploratory analysis was consequently restricted to women with verifiable knee osteoarthritis who were at least 60 years of age, and although this is an obvious limitation, it tried to identify gender-specific impairments and subsets among these cases with affirmed knee osteoarthritis of mild to moderate severity. It was felt important to assess the characteristics of women with established osteoarthritis of one or both knees of some duration but who were generally in good health according to their own estimations. They were specifically examined to elucidate their symptom patterns, perceptions, physical function levels, pain and efficacy cognitions, and perceptions about the condition that may help to advance secondary and/or primary preventive efforts.
The operational definition used to include or exclude potential study candidates required all to have radiographic and clinically verifiable disease as identified by a physician of one or both knees. However, no true comparison as regards the prevailing degree of radiographic damage was available with the exception of a clear distinction between those with unilateral and bilateral disease. This definition may be a confounder though since it does not account for disease duration, nor does it help to identify which cases are hereditary and which are acquired. Age, however, rather than numbers of affected joints, clearly distinguished selected features of the disease associated with disability, regardless of disease duration and extent (See Table 2).

Nonetheless, to support the view that age is only one variable that may influence the natural history of knee osteoarthritis, the extent of pain as reportedly experienced in the last week as assessed prior to taking a walking test battery with the AIMS correlated significantly with the perceived impact of having the disease $(r=0.56, p=0.022)$, as did pain assessed on the visual analogue scale $(\mathrm{r}=0.51$, $\mathrm{p}=0.042$ ) when controlling for age. Other significant predictors of walking (distance) when controlling for age were body mass $(\mathrm{r}=-0.54, \mathrm{p}=0.029)$ and for walking velocity at self-paced speed, plus medical history $(\mathrm{r}=0.49 ; \mathrm{p}=0.052)$, both of which are potentially amenable to intervention. 


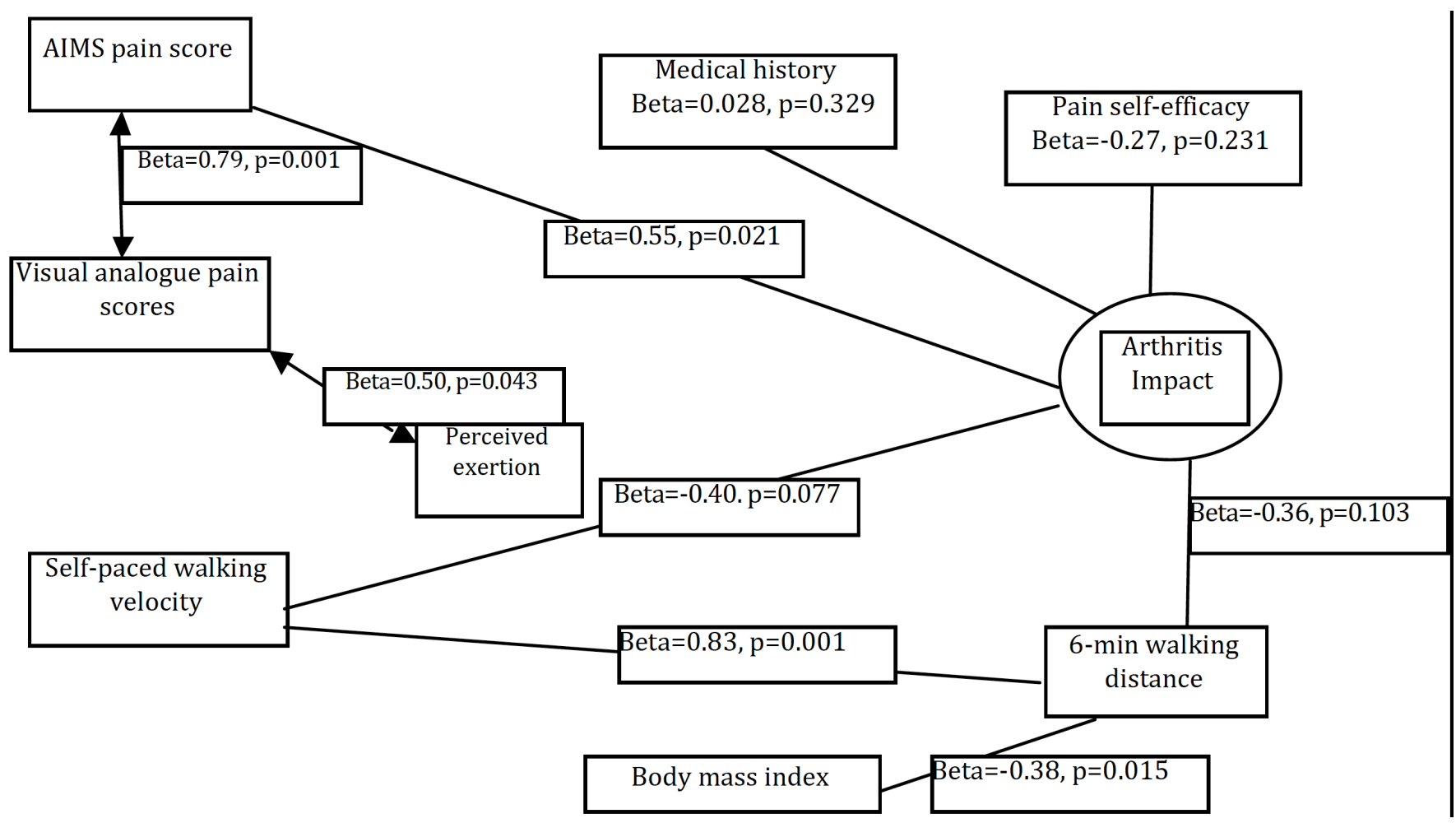

Fig. (1). Diagrammatic representation of salient relationships between measured variables represented by standardized beta $(\beta)$ values and their significance levels.

These findings are important clinically, because while this study did not assess knee muscle strength, a known predictor of function in knee osteoarthritis [6], it is important to note that there was a relationship between the perceived impact of having the disease and locomotor ability as well as knee joint pain, an important determinant of disability [31]. That is, pain, a prognostic factor for worsening of limitations in activities [32] impacted locomotor activity to a significant degree, as did excess body weight, a finding observed by Liu et al. [33]. In addition, those with poorer walking ability perceived their disease affected them more than those with better ambulatory function, a finding also noted by Yildiz et al. [14]. As well, although cardiovascular conditions as a whole did not appear to affect arthritis impact or walking ability, those with cardiac disease histories demonstrated subtle albeit non significant adverse variations in arthritis impact, walking ability scores and pain.

Table 5. Salient partial correlations between AIMS impact scores and key variables when controlling for age $(\mathrm{N}=17)$.

\begin{tabular}{|c|c|c|c|c|c|}
\hline AIMS Impact & AIMS Pain & VAS & SPVel & Pain SE & Weight \\
\hline \hline Partial r, & 0.57 & 0.51 & -0.47 & -0.42 & 0.44 \\
P, & 0.011 & 0.021 & 0.032 & 0.052 & 0.043 \\
\hline
\end{tabular}

Abbreviations: AIMS Impact=Arthritis Impact (0-10); Pain SE=Pain self-efficacy (10100); SPVel=Self-paced walking velocity; VAS=visual analogue scale $(0-10)$

Yet, even though comorbid conditions are common in osteoarthritic samples [19, 32], and increase the likelihood of poor physical function in patients with osteoarthritis [34], many treatment studies for knee osteoarthritis commonly exclude such patients. Alternately, others have focused on examining men and women together [14], and/or hip and knee osteoarthritis together [35]. Moreover, as outlined by Odding et al. [36] the influence of cardiovascular and other diseases and how they interact to diminish function in cases of knee osteoarthritis is relatively unknown even though current information suggests osteoarthritis shares a similar biochemical and inflammatory profile to the metabolic syndrome [37]. Consistent with Miller et al. [39] the present data show a history of cardiovascular disease, including high blood pressure and coronary artery disease, which prevailed in $64 \%$ cases, is very common among women with knee osteoarthritis. Indeed, the presence of one or more of these cardiovascular conditions, has the potential to impact patients both physically speaking and cognitively speaking, perhaps accounting for the presence of depression and related findings of diminished self-efficacy, an important predictor of arthritis self-management in some cases. Those with cardiac disease histories, also appeared to have greater levels of self-reported pain, and slower walking speeds, although this was not significant.

Among other noteworthy findings in this exploratory study was the observation that those with no cardiovascular disease histories had higher body mass indices than those who did not have such a history, and this may imply they are at risk for cardiovascular disease later on, or the early onset of their knee joint osteoarthritis as suggested by related research [37]. Although gait compensations in knee osteoarthritis may be largely neuromuscular, and associated with the extent of pathology, rather than obesity, higher levels of obesity were associated with lower levels of ambulatory function and higher levels of pain, which was consistent with findings by Miller et al. [39]. Moreover, even 
though their functional limitations may be independent of the presence of cardiovascular disease or other disorders [39], given that $82 \%$ of the present cohort were overweight or obese, helping subjects to maintain healthy weights may be key to maximizing ambulatory function because these patients will likely experience elevated cardiac responses during prolonged activities that may further impact their ability to function physically without exertion [20].

Clearly, all subjects in the present study tended to exhibit suboptimal walking speeds and while age accounted partially for this, pain, perceived exertion, and either obesity, and/or the presence of a preexisting cardiac disease appeared to contribute directly or indirectly to the impact of the disease on their ambulatory capacity. Thus, similar to Watanabe et $a l$. who observed that $10 \mathrm{~m}$ gait speed (comfortable and maximum) was significantly inferior in patients with knee osteoarthritis than in controls [38], the present subjects consistently exhibited lengthier gait cycles at self-paced walking speed than one would expect in the average healthy women of the same age. This diminished ability to walk at optimal speeds suggests the oxygen cost per meter traveled is likely to be elevated accordingly among this patient group [20] and can perhaps help to explain the present association between pain, perceived exertion, and walking distance limitations, which were lower on average than those subjects studied by Miller et al. [39].

In particular, the fact that gait cycle duration was significantly related to the perceived impact of their arthritis on their wellbeing, as well as being strongly correlated with pain and pain self-efficacy in this present study suggests that the ability to walk is very salient to the knee osteoarthritis patients' perception of their condition. Consequently, in addition to improving neuromuscular function and reducing high body weights, helping the knee osteoarthritis patient to control pain and improve their self-efficacy for this, as well as treatment related comorbid conditions optimally may help lessen the perceived impact of the condition. However, even though quality of life has recently been linked to ambulatory ability in cases with knee osteoarthritis [38] and the negative effect of comorbid diseases and female gender on health related quality of life among knee osteoarthritis cases has been demonstrated [23], studies in this area are similarly sparse in number, and more are strongly indicated. Indeed, given that middle-aged persons with arthritis are at higher risk for developing mobility and activities of daily living difficulties [40], and middle age women with high body mass indices are at increased risk for knee replacement [41], these factors might be especially valuable as focal points for future exploration and intervention.

At present, due to the limited research in these areas, the most suitable form of conservative therapy may be hard to predict, their efficacy may be impacted adversely, and some modalities such as exercise may even prove risky to apply in a uniform way. Moreover, the most salient factors that impact the presentation of knee osteoarthritis disability outlined in Fig. (1), are often not considered in the context of day to day practice and generic recommendations from wellmeaning organizations and others. Rather than tailoring interventions in accordance with the unique attributes of each patient, universally encouraging exercise for osteoarthritis, along with heat or other modalities, and delivering generic programs to reduce the disability is often recommended instead. Comprehensive approaches applied early on rather than later, which appear crucial because unrelieved or inadequately treated knee osteoarthritis is influenced detrimentally by age, and can lead to heart strain and/or depression, plus obesity that increases knee joint deterioration [34] are rarely forthcoming.

By contrast, given the hypothesized interaction of mechanical factors, psychological and comorbid factors depicted in Fig. (1), it is argued that a significant portion of the female knee osteoarthritis patients' ambulatory impairment may be amenable to intervention using a multidimensional approach. Knee osteoarthritis is a leading cause of osteoarthritis-related impairments in the general United States population [42]. Thus, further research to uncover the importance of other factors that can impact the knee joint osteoarthritis patients'gait capacity, efforts to use advanced technology to detect one or more of these neurological, mechanical and cardiovascular determinants, treating comorbidities [34] and carefully addressing all changeable prognostic factors is strongly indicated in attempts to offset excessive deterioration, and to enhance life quality. More attention to cases with knee osteoarthritis in the highest age categories as well as the highest overweight categories is indicated as well.

\section{CONFLICT OF INTEREST}

The author confirms that this article content has no conflict of interest.

\section{ACKNOWLEDGEMENTS}

Declared none.

\section{REFERENCES}

[1] Altman Rd, Howell DS, Gottlieb NL. New directions in therapy of osteoarthritis. Semin Arthritis Rheum1987; 17(2): 1-2.

[2] Gresham GE, Rathey UK. Osteoarthritis in knees of aged persons, JAMA 1975; 223(2): 168-70.

[3] Sissons HA. Osteoarthritis of the knee: a review. J Rheumatol Suppl 1983: 10: 76-7.

[4] Felson DT, Naimark A, Anderson JL, Kazis W, Castelli RF. The prevalence of knee osteoarthritis in the elderly. Arthritis Rheum 1987; 30(8): 914-8.

[5] Blagojevic M, Jinks C, Jeffery A, Jordan KP. Risk factors for onset of osteoarthritis of the knee in older adults: a systematic review and meta-analysis. Osteoarthritis Cartilage 2010; 18(1): 24-33.

[6] Petterson SC, Raisis L, Bodenstab A, Snyder-Mackler L. Diseasespecific gender differences among total knee arthroplasty candidates. J Bone Joint Surg 2007; 98(11): 2327-33.

[7] Hochberg MC, Lawrence RC, Everett DF, Cornoni-Huntley J. Epidemiologicassociations of pain in osteoarthritis of the knee: data from the national health and nutrition examination survey and the national health and nutritionexamination-i epidemiologic follow-up survey. Sem Arthritis Rheum 1989; 18(4) (Suppl 2): 4-9.

[8] Hadler NM. Osteoarthritis as a public health problem. Clinics Rheum Dis 1985; 11(2): 175-85.

[9] Doyle D. Osteoarthritis. Practitioner 1989; 230(1414): 335-41.

[10] Howell DS. Pathogenesis of osteoarthritis. Am J Med 1986; 80(4B): 24-8.

[11] Felson DT, Gross KD, Nevitt MC, et al. The effects of impaired joint position sense on the development andprogression of pain and structural damage in knee osteoarthritis. Arthritis Rheum 2009; 61(8): 1070-6. 
[12] Glasberg MR, Glasberg JR, Jones RE. Muscle pathology in total knee replacementfor severe osteoarthritis: a histochemical and morphometric study. Henry FordHosp Med J 1986; 34(1): 37-40.

[13] Altman RD, Gray R. Inflammation in osteoarthritis. Clin Rheum Dis 1985;11(2): 353-65

[14] Kaplan D. The epidemiology of osteoarthritis of the knee. J Rheumatol 1983: 9: 87-8.

[15] Yildiz N, Topuz O, Gungen GO, Deniz S, Alkan H, Ardic F. Health-related quality of life (Nottingham Health Profile) in knee osteoarthritis: correlation with clinical variables and self-reported disability. Rheumatol Int 2010; 30(12): 1595-600.

[16] Dekker J, van Dijk GM, Veenhof C. Risk factors for functional decline in osteoarthritis of the hip or knee. Curr Opin Rheumatol 2009; 21(5): 520-4.

[17] Stauffer RN, Chao EY, Györy AN. Biomechanical gait analysis of the diseased knee joint. Clin Orthop Relat Res 1977; (126): 246-55.

[18] Schank JA, Herdman SJ, Bloyer RG. Physical therapy in the multidisciplinary assessment and management of osteoarthritis. ClinTher 1986; 9(Suppl) B: 14-23.

[19] Van Dijk GM, Veenhof C, Schellevis F, et al. Comorbidity, limitations in activities and pain in patients with osteoarthritis. BMC Musculoskeletal Dis 2008; 9(95): 2474-95.

[20] Waters RL, Perry J, Conaty P, Lunsford B, O'Meara P. The energy cost of walking with arthritis of the hip and knee. Clin Orthop Relat Res 1987; (214): 278-84.

[21] Kim I, Kim HA, Seo YI, et al. Tibio femoral osteoarthritis affects quality of life and function in elderly Koreans, with women more adversely affected than men. BMC Musculoskelet Disord 2010; 22; 11: 129 .

[22] Theis KA, Helmick CG, Hootman JM. Arthritis burden and impact are greateramong U.S. women than men: intervention opportunities. J Womens Health (Larchmt) 2007; 16(4): 441-53.

[23] Kauppila AM, Kyllonen E, Mikkonen P, et al. Arokoski JP. Disability in end-stage knee osteoarthritis. Disabil Rehabil 2009; 31(5): 370-80.

[24] Pereira RM, de Carvalho JF, Bonfá E. Metabolic syndrome in rheumatological diseases. Autoimmun Rev 2009; 8(5): 415-9.

[25] Altman R, Asch E, Bloch D, et al. Development of criteria for the classification and reporting of osteoarthritis. Classification of osteoarthritis of the knee. Diagnostic and Therapeutic Criteria Committee of the American Rheumatism Association. Arthritis Rheum 1986; 29(8): 1039-49.

[26] Meenan RF, Gertman PM, Mason JH, Dunaif R. The arthritis impact measurementscales. Further investigations of a health status measure. Arthritis Rheum $1982 ; 25(9)$ : 1048-53

[27] Radloff LS. The CES-D scale: a self report depression scale for research in the general population. Applied Psychol Measurement 1977; 1: 385-401.

[28] Scott J, Huskinsson EC. Graphic representation of pain. Pain 1976; 2(2): $175-84$.
[29] Borg Rating of Perceived Exertion Scale. Available at: http://www.cdc.gov/nccdphp/dnpa/physical/measuring/perceived_e xertion.htm

[30] Lorig K, Chastain RL, Ung E, Shoor S, Holman HR. Development and evaluation of a scale to measure perceived self-efficacy in people with arthritis. Arthritis Rheum 1989; 32(1): 37-44.

[31] Cecchi F, Molino-Lova R, Di Iorio A, et al. Measures of physical performance capture the excess disability associated with hip pain or knee pain in older persons. J Gerontol A Biol Sci Med Sci 2009; 64(12): 1316-24.

[32] van Dijk GM, Veenhof C, Spreeuwenberg P, et al.; CARPA Study Group. Prognosis of limitations in activities in osteoarthritis of the hip or knee: a 3-year cohortstudy. Arch Phys Med Rehabil 2010; 91(1): 58-66.

[33] Liu B, Balkwill A, Banks E, Cooper C, Green J, Beral V. Relationship of height, weight and body mass index to the risk of hip and knee replacements inmiddle-aged women. Rheumatol 2007; 46(5): 861-7.

[34] Kadam UT, Croft PR. Clinical comorbidity in osteoarthritis: associations with physical function in older patients in family practice. J Rheumatol 2007; 34(9): 1899-1904.

[35] Peters TJ, Sanders C, Dieppe P, Donovan J. Factors associated with change inpain and disability over time: a community-based prospective observational study of hip and knee osteoarthritis. Br J Gen Pract 2005; 55(512): 205-11.

[36] Odding E, Valkenburg HA, Stam HJ, Hofman A. Determinants of locomotor disability in people aged 55 years and over: the rotterdam study. Eur J Epidemiol 2001;17(11):1033-41.

[37] Katz JD, Agrawal S, Velasquez M. Getting to the heart of the matter: osteoarthritis takes its place as part of the metabolic syndrome. Curr Opin Rheumatol 2010; 22(5): 512-9.

[38] Watanabe H, Urabe K, Takahira N, et al. Quality of life, knee function, and physical activity in Japanese elderly women with early-stage knee osteoarthritis. J Orthop Surg (Hong Kong) 2010; 18(1): 31-4.

[39] Miller GD, Rejeski WJ, Williamson JD, et al. ADAPT Investigators. The Arthritis, Diet and Activity Promotion Trial (ADAPT): design, rationale, and baseline results. Controlled Clin Trials 2003; 24(4): 462-80.

[40] Covinsky KE, Lindquist K, Dunlop DD, Gill TM, Yelin E. Effect of arthritis in middle age on older-age functioning, JAGS 2008; 56(1): 23-8.

[41] Brennan SL, Cicuttini FM, Pasco JA, et al. Does an increase in body mass index over 10 years affect knee structure in a population-based cohort study of adult women? Arthritis Research Ther 2010; 12(4): R139.

[42] Dillon DF, Rasch EK, Gu Q, Hirsch R. Prevalence of knee osteoarthritis in the United States: arthritis data from the Third National Health and Nutrition Examination Survey 1991-94. J Rheumatol 2006; 33(11): 2271-9. 